\title{
Produção de biomassa e absorção de N e P pela Pfaffia glomerata (Spreng.) Pedersen em função de doses de $\mathrm{N}$ e $\mathrm{P}$ em condições de casa de vegetação
}

\author{
SERRA, A.P.*; MARCHETTI, M.E.; VIEIRA, M.C.; SILVA, M.A.G.; ROSA JUNIOR, E.J.; NASCIMENTO, J.M.; \\ GUEDES, E.M.S. \\ Empresa Brasileira de Pesquisa Agropecuária, Centro Nacional de Pesquisa de Gado de Corte, EMBRAPA- \\ CNPGC, Vila Popular, CEP: 79002-970, Caixa Postal 154, Campo Grande-Brasil *ademar.serra@cnpgc.embrapa.br
}

\begin{abstract}
RESUMO: Com objetivo de avaliar a produção de biomassa e absorção de $\mathrm{N}$ e P pela Pfaffia glomerata em função de doses de $\mathrm{N}$ e P, foi realizado o presente trabalho, em casa de vegetação, utilizando como substrato Latossolo Vermelho distroférrico, coletado na camada de $0-20 \mathrm{~cm}$. 0 delineamento foi inteiramente casualizado com três repetições e os tratamentos foram dispostos em esquema fatorial $5 \times 5$, sendo cinco doses de $\mathrm{N}\left(0,125,250,375\right.$ e $\left.500 \mathrm{mg} \mathrm{vaso}^{-1}\right)$ e cinco doses de $P(0,125,250,375$ e $500 \mathrm{mg}$ vaso-1). As variáveis analisadas foram altura de plantas, massa fresca e seca da parte aérea e radicular e teores de $\mathrm{N}$ e $\mathrm{P}$ na parte aérea e radicular. Os resultados evidenciaram efeito interativo do $\mathrm{Ne} P$, em todas as variáveis analisadas, exceto com relação ao teor de $\mathrm{P}$ na parte aérea e radicular. A produção de biomassa e absorção de $\mathrm{N}$ e $\mathrm{P}$ pela Pfaffia glomerata foi influenciada pelas doses de $\mathrm{N}$ e $\mathrm{P}$ utilizadas no experimento e para a produtividade ótima das características avaliadas, as doses de $\mathrm{N}$ variaram entre 42,9 e 346,01 $\mathrm{mg} \mathrm{vaso}^{-1} \mathrm{e}$ as doses de $\mathrm{P}$ entre os valores de 72,02 e $500 \mathrm{mg} \mathrm{vaso}^{-1}$.
\end{abstract}

Palavras-chave: plantas medicinais, adubação nitrogenada, adubação fosfatada

\begin{abstract}
Biomass production and N and P uptake by Pfaffia glomerata (Spreng.) Pedersen according to $\mathbf{N}$ and $\mathbf{P}$ levels under greenhouse conditions. With the aim of evaluating biomass production and $\mathrm{N}$ and $\mathrm{P}$ uptake by Pfaffia glomerata according to $\mathrm{N}$ and $\mathrm{P}$ levels, the present study was carried out in a greenhouse, using as substrate Oxisol collected at the layer 0 - $20 \mathrm{~cm}$. Experimental design was completely randomized, with three replicates and treatments arranged in $5 \times 5$ factorial arrangement, with five $N$ levels $\left(0,125,250,375\right.$ and $\left.500 \mathrm{mg} \mathrm{pot}^{-1}\right)$ and five $P$ levels $\left(0,125,250,375\right.$ and $\left.500 \mathrm{mg} \mathrm{pot}^{-1}\right)$. The assessed parameters were plant height, shoot and root fresh and dry matter, and $\mathrm{N}$ and $\mathrm{P}$ levels in shoot and roots. Results evidenced an interactive effect of $\mathrm{N}$ and $\mathrm{P}$ for all studied variables, except for $\mathrm{N}$ and $\mathrm{P}$ levels in shoot and roots. Biomass production and $\mathrm{N}$ and $\mathrm{P}$ uptake by Pfaffia glomerata were influenced by the $\mathrm{N}$ and $\mathrm{P}$ levels used in the experiment, whereas the optimal productivity was obtained with $\mathrm{N}$ levels ranging between 42.9 and $346.01 \mathrm{mg} \mathrm{pot}^{-1}$ and $\mathrm{P}$ levels between 72.02 and $500 \mathrm{mg} \mathrm{pot}^{-1}$.
\end{abstract}

Key words: medicinal plants, nitrogen fertilization, phosphorus fertilization

\section{INTRODUÇÃO}

A demanda por plantas medicinais nativas do Brasil tende a aumentar com a descoberta de substâncias que não podem ser obtidas sinteticamente. A utilização dessas plantas tem sido uma prática consagrada em diversas épocas da história humana, sendo encontrados registros em diversas civilizações antigas, onde os únicos medicamentos disponíveis em grande parte eram provenientes dos vegetais. Esta importância perdurou até o século $\mathrm{XX}$, quando do advento da síntese química e fermentação microbiológica que passaram a substituir paulatinamente as plantas medicinais (Bontempo, 1994).

No Brasil, a obtenção de plantas medicinais para uso popular tem ocorrido de forma extrativista e a coleta indiscriminada dessas plantas nativas pode causar sua extinção, a depredação do patrimônio genético e até mesmo engano na utilização das espécies.

Recebido para publicação em 14/05/2009

Aceito para publicação em 05/12/2010

Rev. Bras. PI. Med., Botucatu, v.13, n.3, p.265-270, 2011. 
O conhecimento agronômico sobre essas plantas encontra-se em estágio inicial, quando comparado ao de outras culturas, principalmente no que se refere à sua nutrição mineral, aspecto de elevada importância na obtenção do produto final.

Algumas espécies do gênero Pfaffia estão sendo estudadas por suas propriedades medicinais, atribuídas a substâncias presentes nas raízes. Assim, as raízes de Pfaffia glomerata (Spreng.) Pedersen (Amaranthaceae), bem como de Pfaffia paniculata Kuntze, são de grande interesse comercial, na forma de fito-medicamento e suplementos alimentares, em razão do uso popular como antitumoral, antidiabetes e como tônico afrodisíaco (May et al., 2008), produção de ecdisterona (Fenner et al., 2008; Flores et al., 2009a), $\beta$-ecdisona (Corrêa Junior et al., 2008, Flores et al., 2009b). Segundo Lorenzi (2002), as populações indígenas da Amazônia usam as raízes de Pfaffia glomerata há mais de 300 anos para a cura de ampla variedade de males, como tônico geral, afrodisíaco, calmante e rejuvenescedor.

A Pfaffia glomerata é planta perene, ereta ou semi-ereta, com 0,5 a 2,5 m de altura. Possui raiz tuberosa e, geralmente, bifurcada, como do "ginseng" do Oriente, daí o nome "ginseng brasileiro" ou "ginseng-do-pantanal". As folhas opostas são largas quando jovens, sendo estreitas aquelas próximas das inflorescências. A planta floresce e frutifica de setembro a maio (Pott \& Pott, 1994).

Em solos tropicais o fornecimento de nitrogênio $(N)$ e fósforo $(P)$ pode evitar problemas nutricionais nas plantas, relacionados a esses elementos, devido ao $\mathrm{N}$ ser requerido em grandes quantidades pelas plantas e $\circ \mathrm{P}$ apresentar-se em baixos teores nestes solos, tornando-se limitante ao desenvolvimento das plantas (Malavolta, 2006).

Sendo o $\mathrm{N}$ constituinte de muitos componentes da célula vegetal, incluindo aminoácidos e ácidos nucléicos, a deficiência rapidamente inibe 0 crescimento vegetal (Taiz \& Zeiger, 2004).

No que se refere ao $P$, a cada ano de cultivo há redução dos rendimentos de produção devido ao menor conteúdo de $\mathrm{P}$ a disposição das plantas, como consequência, em parte, da extração desse elemento pelas plantas, sem que haja a reposição em condições de campo (Zhang et al., 2006).

Com base no exposto e considerando-se a necessidade de se conhecer as exigências nutricionais das plantas medicinais, para que correções e reposições adequadas possam ser realizadas, o presente estudo objetivou avaliar a produção de biomassa e a absorção de $\mathrm{N}$ e $\mathrm{P}$ pela Pfaffia glomerata em função das doses de $\mathrm{N}$ e $\mathrm{P}$ em condições de casa de vegetação.

\section{MATERIAL E MÉTODO}

A espécie vegetal Pfaffia glomerata (Spreng.) Pedersen foi cultivada em casa de vegetação, na Faculdade de Ciências Agrárias (FCA), da Universidade Federal da Grande Dourados (UFGD),

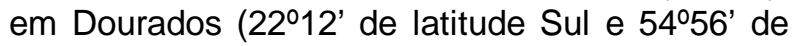
longitude Oeste, altitude de $452 \mathrm{~m}$ ), estado de Mato Grosso do Sul (MS), Brasil.

O solo utilizado como substrato, Latossolo Vermelho distroférrico (Embrapa, 2006), coletado em área de vegetação natural de cerrado, na camada de 0 - $20 \mathrm{~cm}$, seco ao ar, destorroado e passado em peneira com malha de $2 \mathrm{~mm}$, apresenta as seguintes características químicas (Tabela 1).

O experimento foi realizado em vasos, com capacidade para $6 \mathrm{dm}^{3}$ de terra, utilizando-se $6 \mathrm{~kg}$ de terra por vaso. A unidade experimental foi constituída por um vaso contendo uma planta de $P$. glomerata. Testaram-se cinco doses de nitrogênio $(0,125,250$, 375 e $\left.500 \mathrm{mg} \mathrm{vaso}^{-1}\right)$, combinadas com cinco doses de fósforo $(0,125,250,375$ e $500 \mathrm{mg}$ vaso-1). As fontes de nitrogênio e de fósforo utilizadas foram uréia e superfosfato triplo, respectivamente. O delineamento experimental foi o inteiramente casualizado, com os tratamentos dispostos em esquema fatorial $5 \times 5$, com três repetições.

Com base na análise do solo realizou-se a correção da acidez, por meio da utilização de calcário dolomítico finamente moído (PRNT $=100 \%$ ), na dosagem de $16 \mathrm{~g} \mathrm{vaso}^{-1}$ quantidade necessária para elevar a saturação por bases a $70 \%$. Os vasos foram mantidos úmidos, na capacidade de campo, com a utilização de água destilada, incubados por 45 dias.

A semeadura da $P$. glomerata foi realizada em bandejas sem células, no Horto de Plantas Medicinais da FCA/UFGD. O substrato utilizado para a germinação foi uma mistura de solo, areia e casca de arroz carbonizada.

As mudas de $P$. glomerata foram transplantadas para os vasos, 40 dias após a

TABELA 1. Características químicas do solo (Latossolo Vermelho distroférrico) coletado em área de vegetação natural de cerrado, na camada de $0-20 \mathrm{~cm}$.

\begin{tabular}{|c|c|c|c|c|c|c|c|c|c|c|}
\hline $\mathrm{pH}$ & M.O. & $P$ & $\bar{K}$ & $\mathrm{Ca}$ & $\mathrm{Mg}$ & $\overline{\mathrm{Al}}$ & $\mathrm{H}+\mathrm{Al}$ & SB & $\bar{T}$ & $\bar{V}$ \\
\hline $\mathrm{CaCl}_{2}$ & $\mathrm{gdm}^{-3}$ & $\mathrm{mgdm}^{-3}$ & -- & -'-"- & 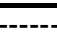 & $--\mathrm{cm}$ & $\mathrm{dm}^{-3}$ & & -- & ---\%--- \\
\hline 3,9 & 16,9 & 1,0 & 0,03 & 0,6 & 0,1 & 1,82 & 9,4 & 0,73 & 10,13 & 7 \\
\hline
\end{tabular}

Laboratório de Fertilidade do Solo da FCA/UFGD, segundo as especificações da Embrapa (1997). 
germinação, ocasião em que foram aplicados os tratamentos com as doses de $\mathrm{N}$ e $\mathrm{P}$, nas doses indicadas anteriormente, sendo o $\mathrm{N}$ aplicado em solução, $1 / 3$ no momento do transplante e 15 dias após, foram aplicados os $2 / 3$ restantes.

Oito meses após o transplante avaliaram-se a altura de plantas e a massa fresca e seca, cortando as plantas rente ao solo e separando-as em parte aérea e radicular. Em seguida, as amostras foram lavadas com água deionizada, colocadas posteriormente para secar ao ar sob papel toalha e conduzidas para pesagem a fim de determinar sua massa fresca. A massa seca foi determinada após a secagem do material em estufa com circulação forçada de ar a $65^{\circ} \mathrm{C}$, até massa constante. O material vegetal foi moído em moinho tipo Willey e acondicionado em sacos plásticos para a realização das análises químicas.

O material vegetal foi moído em moinho tipo Willey e acondicionado em sacos plásticos para a realização das análises químicas.

A determinação do $\mathrm{N}$ foi realizada por digestão sulfúrica pelo método microkjeldhal e nitricoperclórico para a análise dos teores de $\mathrm{P}$, conforme metodologia descrita por Malavolta et al. (1997).

Os dados foram submetidos à análise de variância pelo teste $\mathrm{F}$ e para o caso de diferenças significativas foi realizada a análise de regressão linear múltipla, utilizando-se o aplicativo computacional STATISTICA 8.0.

\section{RESULTADO E DISCUSSÃO}

A altura de plantas foi afetada significativamente $(p<0,01)$ pela interação entre as de doses de $\mathrm{N}$ e de $\mathrm{P}$ da adubação. A superfície de resposta dos dados foi ajustada pela equação de regressão linear múltipla, por meio da qual foi possível estimar os valores máximos, das doses de $\mathrm{Ne} \mathrm{P}$, e a correspondente altura máxima. Obteve-se altura máxima de $1,04 \mathrm{~m}$, com 148,25 g vaso-1 de $\mathrm{N}$ na presença de $424,58 \mathrm{~g} \mathrm{vaso}^{-1}$ de P (Figura 1).

Nos tratamentos com as menores doses de $\mathrm{Ne} \mathrm{P}$ as plantas apresentaram crescimento reduzido (Figura 1), com sintomas visíveis de deficiência de $P$, o que demonstra a necessidade de ajuste de doses de $\mathrm{P}$ e $\mathrm{N}$ adequadas à cultura. Ramos et al. (2000) concluíram que a aplicação de $\mathrm{N}$ e $\mathrm{P}$ em conjunto favorece o crescimento da planta medicinal Pata-devaca (Bauhinia forficata Link.), havendo influência positiva da aplicação das doses de $\mathrm{N}$ sobre a absorção de P. Skrebsky et al. (2008) observaram que, plantas jovens de $P$. glomerata cultivadas em Argissolo Vermelho distrófico arênico apresentaram grande redução no crescimento pela ausência de N, K, S e $P$ na adubação.

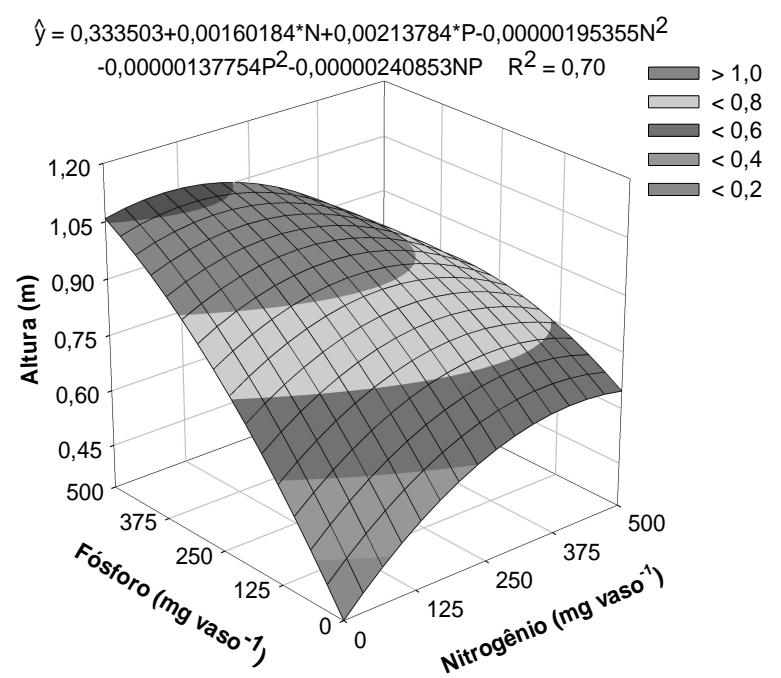

FIGURA 1. Altura de plantas em função de doses de nitrogênio $(N)$, dentro de cada dose de fósforo $(P)$. Dourados-MS, 2004.

O aparecimento de deficiência foi consequência do baixo teor de $P\left(1 \mathrm{mg} \mathrm{dm}^{-3}\right)$ no substrato utilizado para o experimento (Tabela 1), associado com as menores doses de $\mathrm{P}$, dos tratamentos em estudo. No entanto, não foram observados sintomas de deficiência de N. Conforme resultados apresentados por Courtney \& Harrington (2010), solos que apresentam granulometria final com predomínio de óxidos de ferro $\left(\mathrm{Fe}_{2} \mathrm{O}_{3}\right)$ e óxidos de alumínio $\left(\mathrm{Al}_{2} \mathrm{O}_{3}\right)$, como é o caso do Latossolo Vermelho distroférrico, tem uma capacidade maior de retenção do fósforo. Assim, as menores doses de $P$ utilizadas nesse experimento não foram suficientes para superar a capacidade de retenção desses solos, levando ao aparecimento de deficiência de $P$ nas plantas.

$\mathrm{O}$ aumento dos teores de $\mathrm{P}$ elevou $\mathrm{O}$ crescimento vegetativo, com maior altura de plantas (Figura 1) e, por consequência, as plantas sob condições de maior disponibilidade de P, no solo, não apresentaram sintomas visíveis de deficiência de $P$, nas folhas e caules. Quando as plantas apresentam deficiência de $P$, o crescimento torna-se reduzido, e sob condições de deficiência severa as plantas tornamse enfezadas (Epstein \& Bloom, 2006; Malavolta, 2006), sendo tais sintomas apresentados no presente trabalho com a espécie vegetal $P$. glomerata.

Para a variável independente massa fresca da parte aérea, observou-se efeito significativo para a interação $P \times N(p<0,01)$, pelo teste $F$. Ajustou-se uma superfície de resposta aos dados, obtendo a máxima produção estimada de $20,86 \mathrm{~g} \mathrm{vaso}^{-1}$, com a dose de $247,33 \mathrm{mg}$ vaso-1 ${ }^{-1}$ de $\mathrm{N}$ na presença de 500 $\mathrm{mg} \mathrm{vaso}^{-1}$ de $\mathrm{P}$ (Figura 2). 
Verificou-se resposta crescente na produção de massa seca aérea de $P$. glomerata referente às doses de $\mathrm{P}$, sendo a máxima produção estimada igual a $6,06 \mathrm{~g} \mathrm{vaso}^{-1} \mathrm{com}$ a dose de $411,99 \mathrm{mg}$ vaso $^{-1}$ de $P$ e 180,64 $\mathrm{mg} \mathrm{vaso}^{-1}$ de N (Figura 3).

Ramos et al. (2005), trabalhando com menta (Mentha $x$ villosa), em casa de vegetação, com quatro doses de $\mathrm{P}\left(0,5 ; 1,0 ; 10\right.$ e $30 \mathrm{mg} \mathrm{L}^{-1}$ de $\left.\mathrm{P}\right)$, verificaram aumento da produção de massa seca com o incremento das doses de P. Malavolta (2006) relata que a deficiência de $\mathrm{P}$ nas plantas leva a menor

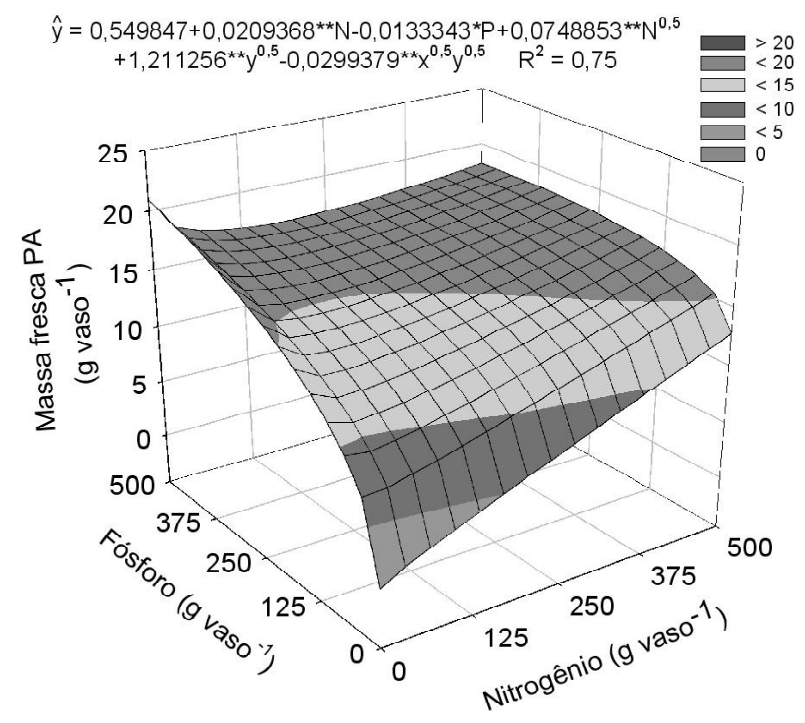

FIGURA 2. Massa fresca da parte aérea (PA) em função de doses de nitrogênio $(N)$, dentro de cada dose de fósforo (P). Dourados-MS, 2004.

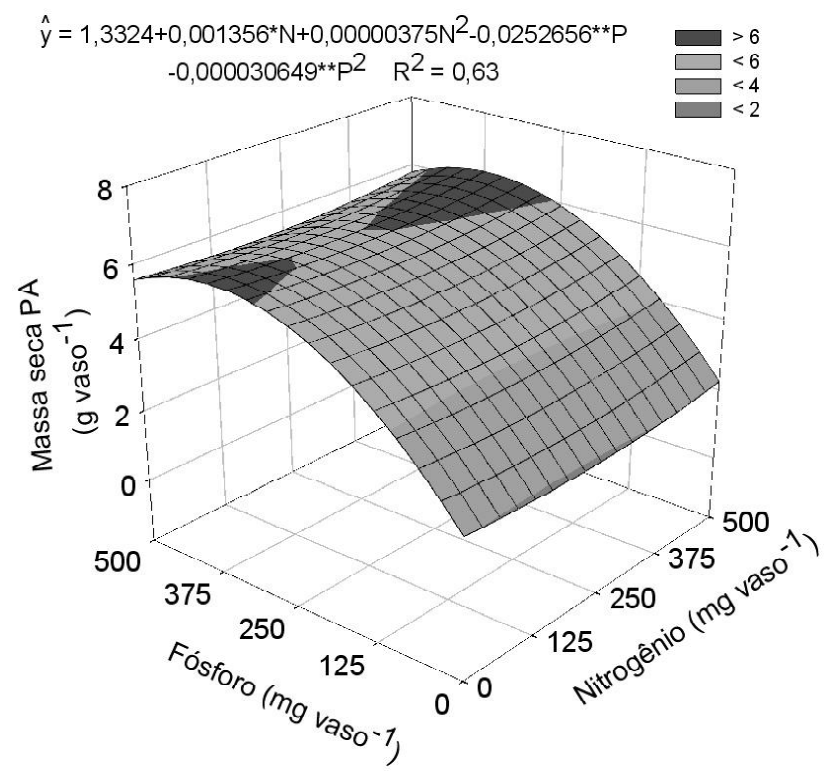

FIGURA 3. Massa seca da parte aérea (PA) em função de doses de nitrogênio $(N)$, dentro de cada dose de fósforo (P). Dourados-MS, 2004. produção de massa seca, levando a menor produtividade. Russowski \& Nicoloso (2003) obtiveram redução na massa seca de parte aérea de $P$. glomerata, quando reduziram a concentração de $P$ no meio de cultura, sendo que tal trabalho foi realizado in vitro.

A massa fresca de raízes apresentou efeito significativo para a interação $(N \times P)$ ao nível de $p<0,01$ pelo teste $F$, sendo possível o ajuste da superfície de resposta aos dados. A dose de $95 \mathrm{mg} \mathrm{vaso}^{-1}$ de N, na presença de $356,27 \mathrm{mg}$ vaso-1 ${ }^{-1}$ de $\mathrm{P}$ foi necessária para se obter a máxima produção de $46,17 \mathrm{~g}$ vaso- $^{-1}$ (Figura 4).

A produção máxima de massa seca de raiz igual a 9,22 $\mathrm{g}$ vaso- $^{-1}$, foi obtida com 42,95 $\mathrm{mg}$ vaso-1 de $\mathrm{N}$ na presença de $363,40 \mathrm{mg}$ vaso-1 de P (Figura 5). Essa resposta na produção de biomassa de raízes, provavelmente deve ter ocorrido devido ao efeito do $P$ em promover a aceleração da formação de raízes e é essencial para seu funcionamento como apoio mecânico e órgão de absorção de água e de íons (Malavolta, 2006; Marschner, 1995).

A produção de massas fresca e seca foi reduzida com as menores dosagens de $P$ utilizadas no experimento, fato esse que corrobora para

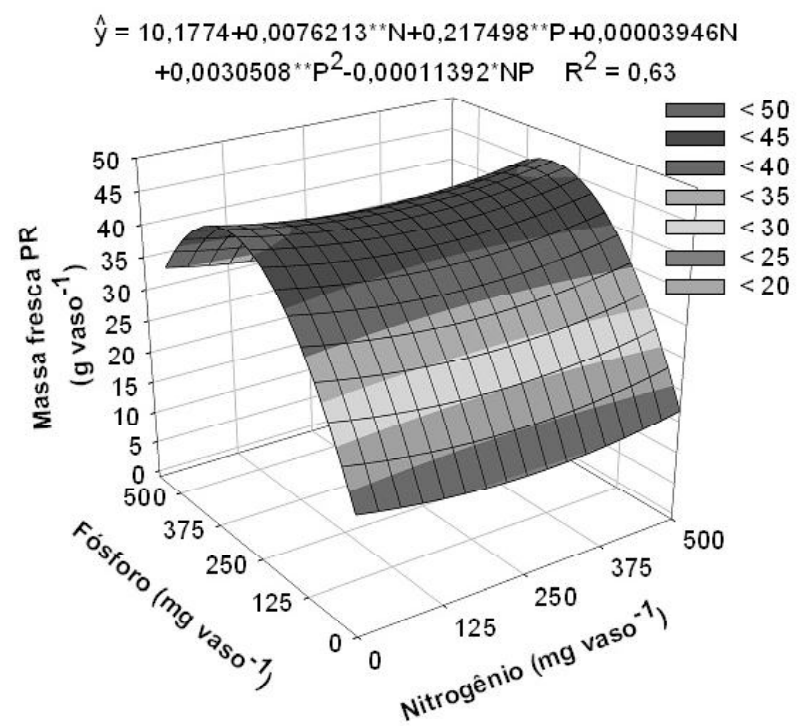

FIGURA 4. Massa fresca da parte radicular (PR) em função de doses de nitrogênio $(N)$, dentro de cada dose de fósforo (P). Dourados-MS, 2004.

comprovação do efeito deletério da deficiência de $\mathrm{P}$ na formação de raízes de $P$. glomerata. No entanto, Skrebsky et al. (2008) relataram que a omissão de P em $P$. glomerata foi aquela que teve menor redução na biomassa radicular, demonstrando que, na deficiência desse nutriente, a planta redireciona a distribuição de fotoassimilados para as raízes. 
O teor de $\mathrm{N}$ na parte aérea das plantas apresentou valor máximo estimado igual a $12,40 \mathrm{~g}$ $\mathrm{kg}^{-1}$ de massa seca, com a dose de 346,01 mg vaso ${ }^{1}$ de $\mathrm{Ne} 72,02$ mg vaso $^{-1}$ de $\mathrm{P}$, estimada pela equação de regressão linear múltipla (Figura 6 ). Pode-se observar nas Figuras 6 e 7, aumento na concentração do $\mathrm{N}$ na planta conforme aumentaram as doses de $\mathrm{N}$ no substrato. $\mathrm{O} N$ é um dos nutrientes que estão presente em maior concentração na planta de $P$. glomerata, exigindo maior absorção, fazendo parte de diversos compostos na planta. Taiz \& Zeiger (2004) relatam que muitos compostos bioquímicos presentes

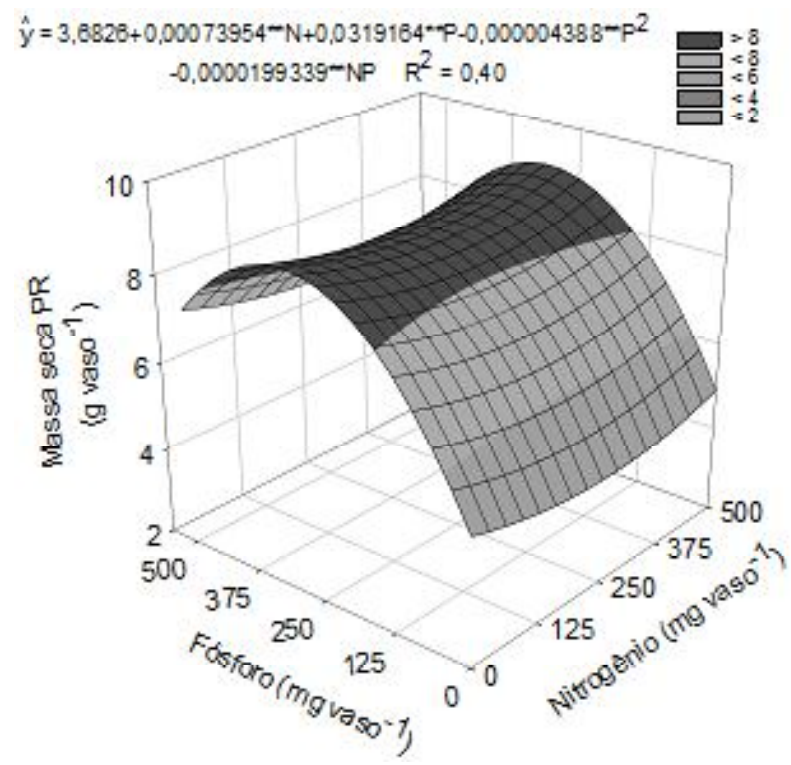

FIGURA 5. Massa seca da parte radicular (PR) em função de doses de nitrogênio $(N)$, dentro de cada dose de fósforo (P). Dourados-MS, 2004.

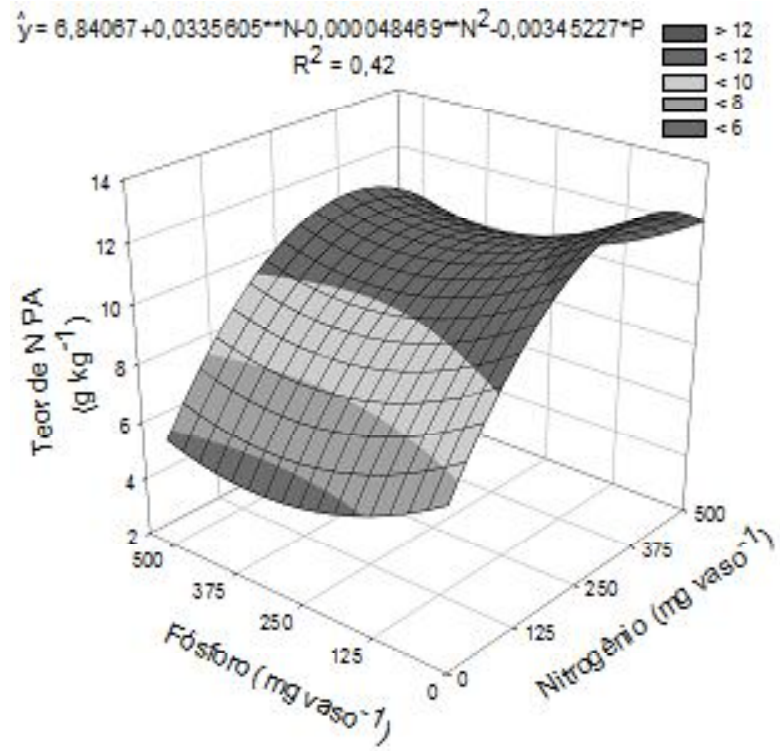

FIGURA 6. Teor de nitrogênio $(\mathrm{N})$ na parte aérea (PA) em função de dose de $\mathrm{N}$, dentro de cada dose de fósforo (P). Dourados-MS, 2004. nas células vegetais possuem $\mathrm{N}$, como os nucleotídeos fosfato e aminoácidos que formam a estrutura dos ácidos nucléicos e proteínas, respectivamente.

Devido à necessidade de $\mathrm{N}$ para a formação de proteínas e outros compostos na planta, o requerimento por esse elemento é elevado (Marschner, 1995), e dessa forma pode-se verificar nas Figuras 6 e 7 resposta crescente dos teores de $\mathrm{N}$ na planta em função do aumento das doses do elemento no substrato. Skrebsky et al. (2008) observaram em plantas de $P$. glomerata que a omissão de $\mathrm{N}$ limitou a absorção e o crescimento dessa espécie, o que se deve a maior exigência de $\mathrm{N}$ tanto pela $P$. glomerata quanto para as demais plantas. A absorção de $\mathrm{N}$ pelas plantas depende da fonte de $\mathrm{N}$ $\left(\mathrm{NO}_{3}-\right.$ ou $\left.\mathrm{NH}_{4}{ }^{+}\right)$disponível ao sistema radicular, pois, dependendo da espécie pode haver melhor absorção quando se utiliza de uma ou outra fonte (Konnerup \& Brix, 2010), devendo esse fato, ser melhor esclarecido em trabalhos futuros para a $P$. glomerata.

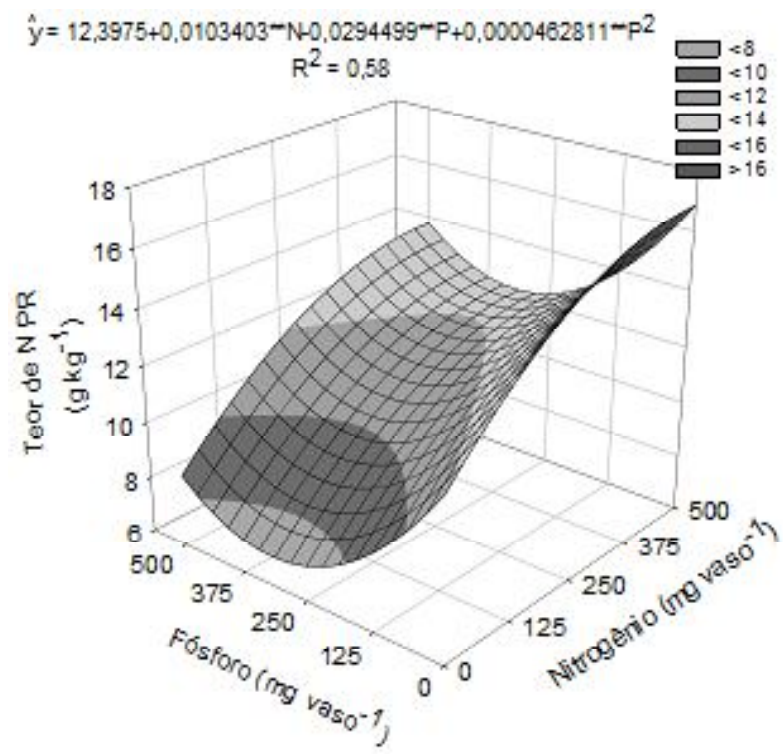

FIGURA 7. Teor de nitrogênio ( $N$ ) na raiz (PR) em função de dose de $\mathrm{N}$, dentro de cada dose de fósforo (P). Dourados-MS, 2004.

O teor de $\mathrm{P}$ nas raízes e na parte aérea não apresentou resposta significativa para as doses de $\mathrm{N}$ e P isoladas aplicadas (Tabela 2). Ramos et al. (2000) observaram que, com o aumento das doses de $\mathrm{N}$ aumentou os teores de $\mathrm{P}$ na planta, havendo dessa forma interação entre os teores de $\mathrm{P}$ e $\mathrm{N}$ em plantas de Pata-de-vaca (Bauhinia forticata Link). A falta de resposta pela planta, aos teores de $P$, possivelmente se deva a menor eficiência de absorção dessa espécie ao P. Skrebsky et al. (2008) relataram que a $P$. glomerata é pouco exigente em $P$ no início do 
desenvolvimento vegetativo, tendo menor absorção desse nutriente. Russowski \& Nicoloso (2003) observaram que a espécie $P$. glomerata apresenta uma exigência nutricional cerca de $30 \%$ maior aos 40 dias do que nos 15 dias, em condições in vitro. Mesmo observando na literatura que a planta aumenta sua eficiência com a idade, possivelmente esse aumento, não seja satisfatório para surtir efeito no teor de $P$ na parte aérea e radicular, com as doses que foram estudadas no presente trabalho.

TABELA 2. Teor de fósforo $(P)$ na parte aérea e radicular de $P$. glomerata em função das doses de nitrogênio (N) e de P. Dourados-MS, 2004.

\begin{tabular}{cccccc}
\hline $\begin{array}{c}\text { Doses de N } \\
\left(\mathrm{mg} \mathrm{vaso}^{-1}\right)\end{array}$ & \multicolumn{5}{c}{ Doses de P } \\
& 0 & 125 & 250 & 375 & 500 \\
\hline \multicolumn{5}{c}{ P na parte aérea } \\
0 & 0,92 & 0,59 & 0,56 & 0,52 & 0,68 \\
125 & 0,73 & 0,87 & 0,85 & 0,91 & 1,18 \\
250 & 0,67 & 0,65 & 1,01 & 0,99 & 0,82 \\
375 & 1,15 & 1,48 & 1,48 & 1,60 & 1,26 \\
500 & 0,63 & 0,47 & 0,67 & 0,51 & 0,60 \\
\hline Média & 0,68 & 0,81 & 0,91 & 0,91 & 0,91 \\
\hline \multicolumn{5}{c}{ P na parte radicular } \\
0 & 0,56 & 0,43 & 0,62 & 0,77 & 0,55 \\
125 & 0,79 & 0,58 & 0,66 & 0,60 & 0,54 \\
250 & 0,83 & 0,46 & 0,57 & 0,45 & 0,48 \\
375 & 0,63 & 0,83 & 0,53 & 0,91 & 1,05 \\
500 & 0,82 & 0,82 & 0,92 & 0,94 & 0,30 \\
\hline Média & 0,73 & 0,62 & 0,66 & 0,73 & 0,58 \\
\hline
\end{tabular}

Pode-se concluir que, a adição de $\mathrm{N}$ no substrato aumentou os teores de $\mathrm{N}$ nas raízes e parte aérea, isso se deve à boa eficiência de absorção e translocação desse elemento pela $P$. glomerata. No entanto, o mesmo não se observou para o $P$, não havendo respostas significativas nos teores de $\mathrm{P}$ na planta.

A produção de biomassa de raiz e absorção de N e P pela Pfaffia glomerata foi influenciada pelas doses de $\mathrm{N}$ e $\mathrm{P}$ utilizadas no experimento, sendo possível relatar que para a produtividade máxima, as doses de $\mathrm{N}$ encontradas estiveram na faixa de 42,95 a $346,01 \mathrm{mg} \mathrm{vaso}^{-1}$ de $\mathrm{N}$ e as doses de $\mathrm{P}$ se mantiveram entre 72,02 a $500 \mathrm{mg}$ vaso $^{-1} \mathrm{de} \mathrm{P}$.

\section{REFERÊNCIA}

BONTEMPO, M. Medicina natural. São Paulo: Nova Cultura, 1994. 584p.

CORRÊA JÚNIOR, C. et al. Sazonalidade na produção de raízes e teor de â-ecdisona em acessos de fáfia. Horticultura Brasileira, v.26, n.2, p.393-7, 2008.
COURTNEY, R.; HARRINGTON, T. Assessment of plantavailable phosphorus in a fine textured sodic substrate. Ecological Engineering, v.36, p.542-7, 2010.

EMBRAPA. Centro Nacional de Pesquisa de Solos. Manual de métodos de análise de solo. 2.ed. Rio de Janeiro: Ed. da EMBRAPA, 1997. 212p.

EMBRAPA. Centro Nacional de Pesquisa de Solos. Sistema brasileiro de classificação de solos. 5 .ed. Rio de Janeiro: Ed. da EMBRAPA, 2006. 169p.

EPSTEIN, E.; BLOOM, A. Nutrição mineral de plantas: princípios e perspectivas. 2.ed. Londrina: Editora Planta, 2006. 401p.

FENNER, R. et al. Hypnotic effect of ecdysterone isolated from Pfafia glomerata (Spreng.) Pedersen. Brazilian Journal of Pharmacognosy, v.18, n.2, p.170-6, 2008.

FLORES, R. et al. Análise de $\beta$-ecdisona em plantas in vivo e in vitro de Pfaffia glomerata (Spreng.) Pedersen, através da Cromatografia em Camada Delgada. Revista Brasileira de Plantas Medicinais, v.11, n.4, p.368-71, 2009a.

FLORES, R. et al. Extração de ecdisterona em raízes de ginseng brasileiro. Ciência Rural, v.39, n.4, p.1223-6, 2009b.

KONNERUP, D.; BRIX, H. Nitrogen nutrition of Canna indica: effects of ammonium versus nitrate on growth, biomass allocation, photosynthesis, nitrate reductase activity and $\mathrm{N}$ uptake rates. Aquatic Botany, v.92, n.1, p.142-8, 2010.

LORENZI, H.; MATOS, F.J.A. Plantas medicinais no Brasil. Nova Odessa: Instituto Plantarum, 2002. 512p. MALAVOLTA, E. Manual de nutrição de plantas. São Paulo: Ed. Agronômica Ceres, 2006. 638p.

MALAVOLTA, E.; VITTI, G.C.; OLIVEIRA, S.A. Avaliação do estado nutricional das plantas: princípios e aplicações. Piracicaba: POTAFÓS, 1997. 319p.

MARSCHNER, H. Mineral nutrition of higher plants. San Diego: Academic Press, 1995. 889p.

MAY, A. et al. Análise de crescimento de fáffia em função do tipo de propagação. Horticultura Brasileira, v.26, n.3, p.375-8, 2008.

POTT, A.; POTT. V.S. Plantas do Pantanal. Corumbá: EMBRAPA-SPI, 1994. 320p.

RAMOS, M.R.C. et al. Influência da aplicação de nitrogênio, fósforo e potássio no crescimento e composição mineral de mudas de Pata-de-vaca (Bauhinia foficata Link). Revista Brasileira de Plantas Medicinais, v.3, n.1, p.79-86, 2000.

RAMOS, S.J. et al. Produção de matéria seca e óleo essencial de menta sob diferentes doses de fósforo. Revista Brasileira Plantas Medicinais, v.8, n.1, p.9-12, 2005.

RUSSOWSKI, D.; NICOLOSO, F.T. Nitrogênio e fósforo no crescimento de plantas de ginseng brasileiro [Pfaffia glomerata (Spreng.) Pedersen] cultivadas in vitro. Ciência Rural, v.33, n.1, p.57-63, 2003.

SKREBSKY, E.C. et al. Caracterização das exigências nutricionais de mudas de Pfaffia glomerata em Argissolo Vermelho distrófico arênico pela técnica do nutriente faltante. Ciência Rural, v.38, n.4, p.989-96, 2008.

TAIZ, T.;ZEIGER, E. Fisiologia vegetal. 3.ed. Porto Alegre: Artmed, 2004. 719p.

ZHANG, Q. et al. Changes in soil phosphorus fractions in a calcareous paddy soil under intensive rice cropping.

Plant and Soil, v.288, p.141-54, 2006. 